\title{
Implications of the development of biosimilars for cancer treatment
}

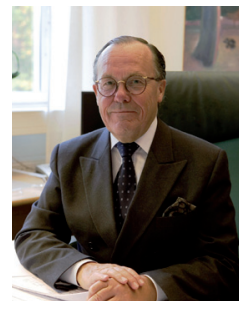

Håkan Mellstedt

Karolinska Institute \& Karolinska University Hospital Solna, Departments of Oncology \& Hematology, CancerCenterkarolinska, SE-171 76 Stockholm, Sweden m Tel.: +46851 774308 = Fax: +46 8318327 n. hakan.mellstedt@karolinska.se
"Biosimilars will undoubtedly have a great impact on cancer treatment as these protein drugs, to a lower cost, will increase the utility of biopharmaceuticals for a large patient population and thereby improve the overall outcome for cancer patients."
By the use of recombinant technologies, a variety of therapeutic proteins have allowed biopharmaceuticals to become important options for a variety of oncology indications. However, a number of patent expirations for biopharmaceuticals have prompted the development of alternative versions of biological products called biosimilars [1].

Biosimilars are agents that are similar but not identical to the innovator product. Characteristics of therapeutic proteins are closely related to the manufacturing process, which cannot be duplicated. Thus, biosimilars are unique molecules and not generic versions of the innovator biopharmaceuticals.

The primary and secondary structures of a biosimilar should be the same as the reference product [2], as should the biological functions, shown in preclinical testing systems, indicating the same mechanisms of action.

In clinical trials with a rather small number of patients and short observation periods, biosimilars have shown similar clinical effects to the reference product. Erythropoietin biosimilars have been shown to have the same effects on hemoglobin concentration in chronic renal failure and chemotherapy-induced anemia as the reference product. Similarly, granulocyte colony-stimulating factor (G-CSF) biosimilars have a similar effect as the innovator drug on chemotherapy-induced neutropenia and CD34 ${ }^{+}$ stem cell mobilization in healthy donors.

However, the tertiary structure of the biosimilar may be different from that of the innovator drug. Identity, similarity and/or difference in tertiary structures are difficult to prove. Such differences may have consequences, especially with regard to immunogenicity but maybe also to biological functions. These differences may not be seen until a substantial number of patients have been treated and followed up over an extended time period, longer than that required for registration. Postmarketing programs are extremely important for biologicals, much more than for small molecules.

\section{${ }^{6}$ Postmarketing programs are extremely important for biologicals, much more than for small molecules."}

Antibodies induced against a biopharmaceutical may interact with the corresponding endogenously produced protein and cause severe side effects (e.g., pure red cell aplasia). These effects might only be seen in a minority of patients but they are still of clinical significant importance. Immunogenicity is not only related to characteristics of the protein molecule but also to the formulation and route of administration. Recently, the same erythropoietin biosimilar was shown to induce a few cases of pure red cell aplasia when switched from intravenous to subcutaneous administration [3]. Repeated administrations of growth factors over a long time period, such as erythropoietin in cancer-related anemia and fatigue, may increase the risk of antibody induction, which has not been possible to observe in the registration studies.

Another problem is extrapolation [1], which may be linked to the nonidentity of the biochemical structures of the biosimilar compound compared with the innovator drug, to indications approved for the innovator drug even though the biosimilar drug has not been formally tested for the given indications. Extrapolation is probably acceptable. However, there might be special concerns, as the use of G-CSF in healthy donors for stem cell

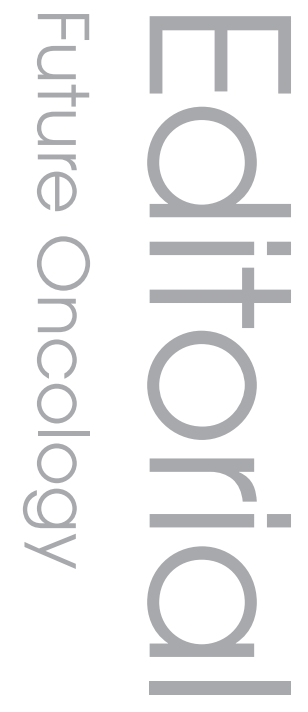


mobilization. A careful long-term monitoring of healthy donors treated with G-CSF biosimilars should be mandatory with regard to side effects such as immunogenicity and on normal WBC functions and leukemogenesis. The quality of the mobilized stem cells is another important issue to monitor. In chronic neutropenia, efficacy and immunogenicity are important to follow. Another concern is the use of biosimilars in pediatric oncology.

Automatic substitutions are allowed for chemical generics. In principle, this rule could also be applied to biosimilars. However, authorities in most European countries do not recommend or allow automatic substitutions of biosimilars [1]. This is an interesting standpoint, indicating that the authorities are aware of the fact that biosimilars are not identical drugs and that different biosimilars should not be mixed. Side effects and clinical inferiority of biosimilars may not be possible to trace if automatic substitution is allowed; important clinical information on individual biopharmaceuticals may be lost.

Patents on monoclonal antibodies (MAb) will soon expire, and a huge market for MAb biosimilars (bMAb) is expected [4]. Compared with growth factors, MAb are much more complex in structure, and similarities/comparability to the innovator drugs will be more difficult to prove than for growth factors. MAbs have multifunctional mechanisms of action, and their biochemical characteristics can have a significant effect on biological functions.

It will be difficult to duplicate the amino acid sequences of the complimentarity-determining regions I-III defining the antigen-binding site. The affinity-binding constant will differ. It will not be easy to copy the biological active part of the immunoglobulin molecule, the Fc-part, with its complex amino acid sequences and carbohydrate contents determining antibodydependent cellular cytotoxicity and complement-dependent cytolysis. However, it may not be necessary to have identical amino acid sequences within, for example, the framework regions or in all parts of the constant region of the immunoglobulin molecule to have a comparable MAb. Guidelines should be adopted regarding this issue where we do not have detailed knowledge.

The multifunctionality of MAbs and the uncertainty of the relative contributions of various effector functions, such as signaling inhibition and/or apoptosis induction, antibody-dependent cellular cytotoxicity and complement-dependent cytolysis, leading to the tumor cell death of a MAb should make it difficult to produce a $\mathrm{bMAb}$ with similar claims to those of the innovator drug.

Clinical surrogate end points such as increase in hemoglobin concentration induced by erythropoietin and white blood cell count by G-CSF are easy to establish. Biomarkers for clinical efficacy of a MAb in oncology are not available. Randomized clinical studies are probably required measuring both response rates and time to progression or overall survival to compare favorably with the innovator drug. All these obstacles will force biopharmaceutical companies to accomplish extensive developmental programs for $\mathrm{BMAb}$ and the authorities to proceed with care and attention. What are urgently needed are biomarkers that relate to clinical efficacy in order to reduce the extent of clinical trials required. Development of bMAb might also be facilitated by sensitive modeling systems.

Immunogenicity has been a major concern for growth factor biosimilars. However, this will probably not be the case for bMAb. Antibodies against biosimilar monoclonal antibodies will only reduce efficacy, but no other severe side effects are expected as therapeutic monoclonal antibodies do not have a corresponding endogenously produced protein.

"Antibodies against biosimilar
monoclonal antibodies will only reduce
efficacy, but no other severe side effects
are expected as therapeutic monoclonal
antibodies do not have a corresponding
endogenously produced protein."

Economics is a driving force for the introduction of biosimilars. There are great hopes for cost reductions. However, these reductions will not be in the range of small chemical molecule generics. It is realistic to expect $30 \pm 10 \%$ lower costs for biosimilars compared with the reference products. From a national economic point of view, such a level of price reduction is of great importance and should tremendously influence the use of these expensive antitumor drugs and also facilitate a rational clinical development. Hopefully, many more investigatorsponsored studies will be performed, which might lead to a better and more optimal use of biopharmaceuticals in oncology.

The biopharmaceutical market will be characterized by price competition, even when there are a limited number of players for a given product. This holds for both original and biosimilar 
protein drugs. The market for biosimilars is huge, particularly when biosimilars enter the US market. The annual market value is expected to be more than US $\$ 20$ billion in 5 years time."

Biopharmaceuticals, especially biosimilars, will be important drugs and represent a substantial part of the health economy. To demonstrate value for money, it is important that health economics are involved early in the developmental process of biopharmaceuticals and biosimilars. It is also important that health professionals, such as physicians, pharmacists and other healthcare decision-makers, are aware of the advantages but also the potential drawbacks of biosimilars in order to apply an optimal use of these novel innovative drugs.

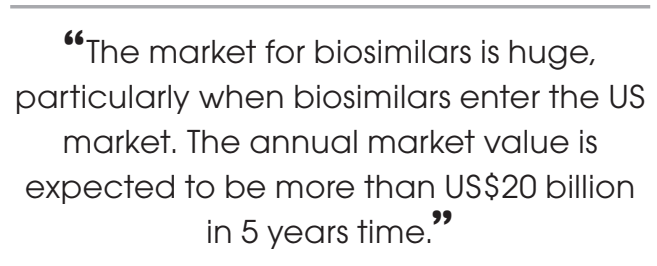

The future will bring rapidly increasing access to various biopharmaceuticals in oncology and will require a novel competence among healthcare providers, as well as care and attention from the authorities. It will require an intense dialog between healthcare professionals and the industry, and the inclusion of health economics in the evaluation process.

\footnotetext{
Financial \& competing interests disclosure

The author has been scientific advisor to Amgen and Sandoz and receives a research grant from Merck GmbH for developing of cancer vaccines. The author has no other relevant affiliations or financial involvement with any organization or entity with a financial interest in or financial conflict with the subject matter or materials discussed in the manuscript apart from those disclosed.

No writing assistance was utilized in the production of this manuscript.
}

\section{Bibliography}

1. Mellstedt H, Niederwieser D, Ludwig H: The challenge of biosimilars. Ann. Oncol. 19, 411-419 (2008).

2. Brockmeyer C, Seidl A: Binocrit: assessment of quality, safety and efficacy of biopharmaceuticals. EJHP Practice 15, 34-40 (2009).

3. Head of Medicine Agencies. Monthly Report. Erythropoetin - Risk of Pure Cell Aplasia, Issue No. 0912. EMA/816248/2009 (2010).

4. Reichert JM, Beck A, Iyer H: European Medicines Agency workshop on biosimilar monoclonal antibodies: July 2, 2009, London, UK. Landes Biosci. $m A b s$ 1(5), 394-414 (2009). 